\title{
Characterizing the emergence and persistence of drug resistant mutations in HIV-1 subtype C infections using 454 ultra deep pyrosequencing
}

\author{
Vijay Bansode ${ }^{1}$, Grace P McCormack ${ }^{1}$, Amelia C Crampin ${ }^{2,3}$, Bagrey Ngwira ${ }^{2,3}$, Ram K Shrestha ${ }^{4}$, Neil French ${ }^{3,5}$,
} Judith R Glynn ${ }^{3}$ and Simon A Travers ${ }^{4^{*}}$

\begin{abstract}
Background: The role of HIV-1 RNA in the emergence of resistance to antiretroviral therapies (ARTs) is well documented while less is known about the role of historical viruses stored in the proviral DNA. The primary focus of this work was to characterize the genetic diversity and evolution of HIV drug resistant variants in an individual's provirus during antiretroviral therapy using next generation sequencing.
\end{abstract}

Methods: Blood samples were collected prior to antiretroviral therapy exposure and during the course of treatment from five patients in whom drug resistance mutations had previously been identified using consensus sequencing. The spectrum of viral variants present in the provirus at each sampling time-point were characterized using 454 pyrosequencing from multiple combined PCR products. The prevalence of viral variants containing drug resistant mutations (DRMs) was characterized at each time-point.

Results: Low abundance drug resistant viruses were identified in 14 of 15 sampling time-points from the five patients. In all individuals DRMs against current therapy were identified at one or more of the sampling time-points. In two of the five individuals studied these DRMs were present prior to treatment exposure and were present at high prevalence within the amplified and sequenced viral population. DRMs to drugs other than those being currently used were identified in four of the five individuals.

Conclusion: The presence of DRMs in the provirus, regardless of their observed prevalence did not appear to have an effect on clinical outcomes in the short term suggesting that the drug resistant viral variants present in the proviral DNA do not appear to play a role in the short term in facilitating the emergence of drug resistance.

Keywords: HIV-1, Drug resistance, Subtype C, Malawi, Ultradeep sequencing, Proviral DNA

\section{Background}

While the role of HIV-1 RNA in the emergence of resistance to highly active antiretroviral therapy (HAART) has been widely documented, less is known about the role of historical viruses stored in the proviral DNA. Following treatment interruption, latent viruses stored in the provirus have been shown to be responsible for the rapid rebound of viral load following treatment interruption [1-3]. However, Palmisano and colleagues suggested that the mutational archive stored in proviral DNA remains

\footnotetext{
* Correspondence: simon@sanbi.ac.za

${ }^{4}$ South African National Bioinformatics Institute, University of the Western, Cape, Bellville, South Africa

Full list of author information is available at the end of the article
}

unchanged during HAART [4] with higher levels of DRMs observed in RNA extracted from circulating virus than those present in the provirus [5]. The suggested reason for these discrepancies is that standard bulk sequencing cannot fully access the spectrum of viral variants stored in the proviral DNA [5] where resistant viruses may be present in low abundance.

Recent research on HIV-1 in Karonga District, Malawi has shown that of the $40 \mathrm{HIV}-1$ subtype $\mathrm{C}$ infected individuals on HAART that we tested, $14 \%$ contained drug resistance mutations (DRMs) in the reverse transcriptase (RT) gene of viruses stored in the provirus [6] with clonal sequencing of latent viruses showing discrepancies in the presence and prevalence of DRMs in the

\section{Biomed Central}


proviral DNA [7]. These discrepancies further support the limitations of bulk sequencing for determining drug resistance in viruses contained within the proviral DNA. Traditionally, methods such as single genome amplification and real-time PCR have been used to identify the presence of low abundance viral variants $[8,9]$. We, and others, have shown the importance of clonal sequencing in revealing the spectrum of viral variants present within an individual [6]. However cloning and other traditionally used methods are labour intensive, expensive, time consuming and/or restricted to the detection of single variants. The advent of next generation sequencing platforms means that clinically relevant low abundance drug resistant HIV variants can be detected to prevalences as low as $1 \%$ of the viral population [10-16]. While several studies have found correlations between the presence of low abundance drug resistant viruses and clinical outcomes this has not always been the case $[17,18]$. To our knowledge, no studies have been undertaken to use ultra deep pyrosequencing (UDPS) to quantify the prevalence of low abundance drug resistant viral variants in the proviral DNA. Here, we undertake such a study and endeavor to correlate the presence/absence of such viruses with treatment outcome.

\section{Methods}

\section{Sample selection}

Samples were collected from five patients recruited to an antiretroviral cohort study between 2007 and 2009 from an ART clinic in northern Malawi. Antiretrovirals have been available in the District since 2005 and in this clinic since 2006. All patients have been started on a fixed dose combination of stavudine, lamivudine and nevirapine on the basis of clinical staging or CD4 count. Previous work identified discrepancies or ambiguities in the presence/absence of drug resistant mutations in viral sequences from samples collected from these five individuals at sequential time-points during treatment [6]. Fifteen samples representing an average of three sampling time-points from five of these patients were selected for subsequent analysis using ultra-deep pyrosequencing in this current study (Table 1).

\section{Ultra deep pyrosequencing (UDPS)}

DNA extraction and nested PCR was performed as previously described [6] with the second round PCR primers modified to include the A and B adaptors necessary for 454 sequencing. Each second round primer also included a unique sequence tag (MID) to enable us to distinguish between samples from the multiplexed sequencing reaction. The region targeted for amplification was 760 nucleotides in length covering amino acid positions 13 through 257 of reverse transcriptase (HXB2 numbering). Temperature gradient PCRs identified $57.5^{\circ} \mathrm{C}$ as the optimum annealing temperature for all secondary PCR reactions with the extended primer sequences for UDPS. Considerable effort was invested into optimizing the PCR amplification approach to account for potential over-amplification of a subset of viral variants present in the quasispecies. For each sample the final PCR strategy involved performing ten first round $100 \mu \mathrm{l}$ PCR reactions. $5 \mu \mathrm{l}$ of the product of each primary PCR was used as starting material for two separate secondary PCR reactions, generating a maximum of 20 secondary amplification reactions from each sample. PCR products were quantified on $1 \%$ agarose gels in comparison with the Hyperladder 1 molecular marker (Bioline). The 20 PCR products generated from each DNA sample were then mixed in equimolar amounts and an aliquot of the mixed products arising from each DNA sample were then electrophoresed side by side for comparison and further confirmation of quality and quantity. PCR products from all samples were then further pooled in equimolar amounts. Amplicon sequencing was performed on one half of a picotiter plate using a 454 Genome Sequencer FLX by LGC Genomics (Germany).

\section{Data cleaning and analysis}

Sequence reads from each individual sample were separated based on their MIDs and subsequent quality control and analysis was performed independently for the reads corresponding to each sample. Next, the data were trimmed using the modified-Mott algorithm implemented in Geneious 5.4.3 [19] with a 0.01 error probability limit. Initially the identity of the resulting reads to their respective consensus sequence was determined using the BLAST-like word (k-mer) matching approach implemented in Segminator 1.3.2 [20] with a word size of five and a read quality of two. Reads with significant identity were aligned to the consensus sequence excluding any reads less than 22 nucleotides in length, the dataset was translated to amino acids and the frequency of amino acids at each position was determined using Segminator 1.3.2 [20].

The prevalence of drug resistant mutations (DRMs) was determined for each sample with three categories of DRMS identified. Firstly, DRMs detected in less than 1\% of the reads sequenced from the amplified viral population for that position were discounted to account for potential errors due to the error rate of PCR and UDPS. The second category of mutations consisted of those with a prevalence greater than $1 \%$ and less than $20 \%$, corresponding to those that cannot be determined using consensus sequencing. The final category consisted of those DRMs with prevalence greater than $20 \%$, comprising mutations that can, in theory, be observed using traditional consensus sequence genotyping. Given that the number of reads that were sequenced at each 
timepoint in a patient were unlikely to be exactly the same, we implemented a statistical approach for prevalence calculations as opposed to using a rigid fixed cutoff. For every drug resistant mutation, the $95 \%$ confidence interval from the binomial sampling distribution was calculated for the observed frequency taking into account the number of reads sequenced at that timepoint. The upper 95\% confidence interval was used as a cutoff for the allocation of DRMs into both the greater than $1 \%$ and less than $20 \%$ category and the greater than $20 \%$ category.

Recent work has described HIV drug resistance mutations that are located within, or adjacent to, homopolymers in the HIV Pol gene [21]. Given that one of the known shortcomings of the 454 pyrosequencing approach is that it can cause false insertions or deletions in homopolymeric tracts, we undertook an approach to verify if drug resistance mutations at such homopolymers were genuine DRMs or arose as the result of sequencing error. We have developed a novel tool called rapid amplicon mapping in codon space (RAMICS) that uses hidden markov models to map next generation sequencing reads to a reference sequence in codon space, thus generating a biologically relevant alignment (Wright et al., in prep). This approach identifies the presence of hompolymer runs in data and considers them in the mapping process, thus accounting for the possible variances in length as a result of sequencing error. Using RAMICS we confirmed whether resistance calls at DRMs located at homopolymer tracts in the sequence data for all of the datasets were biologically relevant or had arisen as a result of sequencing error.

Permission for the study was received from the National Health Sciences Research Committee, Malawi, and the Ethics Committee of the London School of Hygiene and Tropical Medicine, UK. Written informed

Table 1 Samples subjected to ultra-deep pyrosequencing in this study

\begin{tabular}{|c|c|c|c|c|c|}
\hline Patient Number & Sampling Time-point & NRTI mutations & NNRTI mutations & Primary PCR & Secondary PCR \\
\hline \multirow[t]{4}{*}{ Patient 2} & Baseline & & & 9 & 9 \\
\hline & 8 Months & & K103KN & & \\
\hline & 11 Months & & Y181C & 10 & 20 \\
\hline & 14 Months & & & 3 & 20 \\
\hline \multirow[t]{7}{*}{ Patient 32} & Baseline & & V90I & 6 & 9 \\
\hline & 7 Months & $\mathrm{T} 215 \mathrm{ST}$ & & 2 & 20 \\
\hline & 8 Months & M184I & & & \\
\hline & 14 Months & & & & \\
\hline & 15 Months & & G190AE & & \\
\hline & 16 Months & & V108AV & 3 & 20 \\
\hline & 24 Months & & & 2 & 20 \\
\hline \multirow[t]{3}{*}{ Patient 42} & Baseline 1 (2008) & & & 2 & 7 \\
\hline & Baseline 2 (2009) & V118IV & & 5 & 20 \\
\hline & 5 Months & V118I & & & \\
\hline \multirow[t]{3}{*}{ Patient 45} & Baseline & & V106I, E138A, G190A & 9 & 20 \\
\hline & 8 Months & & V106I, E138A, G190A & 10 & 20 \\
\hline & 13 Months & & Sequence Failed & 10 & 20 \\
\hline \multirow[t]{4}{*}{ Patient 76} & Baseline & & V90I, Y181CY, H221HY & 10 & 20 \\
\hline & 3 Months & & & & \\
\hline & 6 Months & & Y181C & 10 & 15 \\
\hline & 9 Months & & Sequence Failed & 10 & 19 \\
\hline
\end{tabular}

Consensus sequencing of reverse transcriptase from five patients had previously revealed discrepancies in the presence/absence of DRMs [6,7]. Baseline describes the sampling time-point prior to ART initiation with subsequent samples being described as the number of months post ART initiation. Mutations shown in the table are those observed in the consensus sequencing of each sampling time-point with DRMs to current therapy marked in bold. The sample time-point names for samples selected for subsequent ultra-deep pyrosequencing are marked in bold and the success rate for multiple PCRs for these samples are also shown. The remaining six samples were not chosen due to difficulty/failure with PCR amplification or consensus sequencing. 
consent for participation in the study was obtained from all participants.

\section{Results}

\section{DNA amplification and UDPS data cleaning}

Despite considerable effort, PCR amplification was not uniformly successful for all DNA samples. Secondary PCR was successful from ten primary PCRs for six of the 15 samples with all 20 secondary PCR reactions being successful in four of these (Table 1). Amplification from primary PCRs was less successful for the remaining samples ranging from positive secondary amplification from two to nine first round reactions (Table 1). In instances where secondary PCRs were unsuccessful from primary reactions, additional secondary PCRs were performed from those primary PCR products that did yield amplification, in order to maximize diversity (Table 1). Despite multiple attempts to obtain 20 secondary PCR amplifications, fewer than 10 secondary products were obtained from the baseline sample from patients 2, 32 and 42. Although secondary amplification was successful in all 20 reactions from the $2^{\text {nd }}$ baseline sample from patient 42 , the amplification was very weak compared to secondary amplifications from other patients. Similar results were obtained from $16^{\text {th }}$ month from Patient 32 and $13^{\text {th }}$ month sample from patient 45 .

\section{Deep sequencing and data cleaning}

454 sequencing resulted in a total of 372,169 reads of which 1038 could not be sorted on the basis of their MID. Of the remaining 371,131 reads the number of sequences representing each of the 15 samples ranged from 12,926 to 38,514 (Additional file 1: Figure S1A). Quality trimming was employed with a 0.01 error probability limit meaning that each trimmed read has a minimum average accuracy greater than $99 \%$. This trimming step did not result in the removal of any reads (as no minimum length requirement was imposed in this step), however the average length of all reads was significantly decreased from 541 for reads before trimming to 176 for reads following trimming (Additional file 1: Figure S1B).

The number of quality trimmed reads we attempted to map to the template sequence ranged from 12,925 to 38,459 with reads being excluded from the mapping procedure as a result of falling below the minimum read length score (22 nucleotides) or due to low identity to the template sequence. Between 677 and 3007 reads per patient were excluded based upon the read length cutoff (Additional file 1: Figure S1B) with a range of 1324 and 14,609 reads per patient excluded because of low identity to the template. Three samples (Patient 45 at 13 months, patient 32 at 16 months and patient 42 baseline 2) had large numbers of reads excluded during the mapping process with $43 \%, 61 \%$ and $65 \%$ percent of reads removed respectively. In each of these cases the majority of reads were removed due to low identity to the template sequence. Further analysis of the excluded reads from these individuals showed that a large proportion of them mapped to regions throughout the HIV genome outside of the region of interest. Sequencing coverage towards the centre of each amplicon was significantly less than at either the $5^{\prime}$ or $3^{\prime}$ end of the amplicon.

\section{Prevalence of drug resistance mutations identified with deep sequencing}

In all samples the vast majority (between $74 \%$ and 96\%) of drug resistance mutations identified by deep sequencing were detected at prevalence levels less than $1 \%$ of the sequenced viral population (Figure 1A) and were, thus, excluded from any subsequent analysis. For the remaining DRMs, we assessed the effect of sequencing error at homopolymeric regions and, in all instances, we found that sequencing error was not responsible for the resistance calls indicating that they are, in fact, genuine. An average of $11 \%$ of observed DRMs (range 2.5$21.21 \%$ ) were detected at prevalence levels between $1 \%$ and $20 \%$ of the sequenced viral population with an average of $6 \%$ (range $0-12.5 \%$ ) identified at greater than $20 \%$ prevalence in the sequenced viral population (Figure 1A and Table 2).

UDPS identified an additional 32 DRMs (17 against current therapy) that had not been observed by consensus sequencing (Table 2). The vast majority of these (26) were observed at a prevalence between $1-20 \%$ of the sequenced viral population (Table 2). Bulk consensus sequencing was not successful for the final samples from patients 45 and 76 (Table 1), however UDPS identified DRMs at both 1-20\% (9 DRMs, 4 against current therapy) and greater than $20 \%$ prevalence (6 mutations, 2 against current therapy) within the sequenced viral population (Table 2). Of the 15 DRMs (five to current therapy) seen in bulk sequencing of samples subjected to UDPS (Table 1), 10 of these (four against current therapy) were also observed in the UDPS sequencing with the vast majority of these ( 8 of the 10) being seen at a prevalence greater than $20 \%$ in the sequenced viral population (Table 2). The two DRMs observed at less than $20 \%$ of the sequenced viral population were V90I (9.5\% prevalence) and $\mathrm{H} 221 \mathrm{Y}$ (7.7\% prevalence) in the baseline samples from patients 32 and 76 respectively. Three DRMs identified in bulk sequencing (Y181C in patient 2 at 11 months and T215ST and V108AV in patient 32 at 7 and 16 months respectively) were not observed at any level of prevalence in UDPS. DRMs V118I and E138A, from patients 42 (baseline 2) and 45 (8 months) respectively, were observed in the UDPS data but sequence coverage at these positions was below the cutoff. 


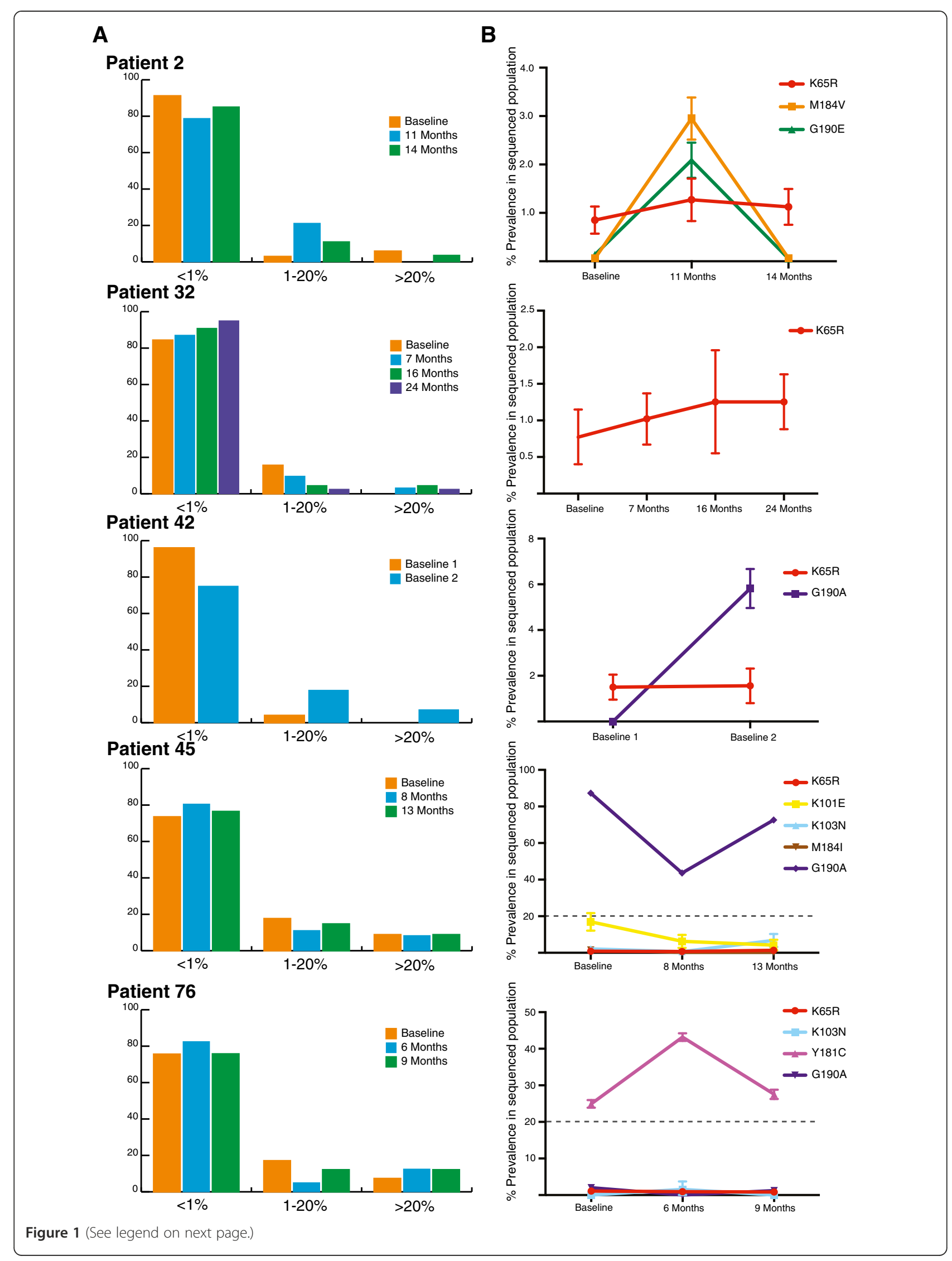


(See figure on previous page.)

Figure 1 Observed DRMs at all sequenced sampling time-points for each patient. Panel $\mathbf{A}$ shows the percentage of DRMs expressed as in each prevalence category for each patient. DRMs observed at less than $1 \%$ of the amplified and sequenced viral population were discounted from further analysis in this study to account for potential errors due to the error rate of PCR and UDPS but are shown in this figure for comparison purposes. Panel B shows the prevalence of DRMs present in the amplified and sequenced viral population against the patients' current therapy at each of the sampling time-points. For each prevalence point, the upper and lower 95\% confidence intervals from the binomial sampling distribution are shown as error bars. DRMs with a prevalence less than 1\% of the amplified and sequenced viral population are not included.

Of the mutations detected at greater than $1 \%$ prevalence by UDPS against current $1^{\text {st }}$ line therapy, only K65R was observed, albeit at varying levels, in all of the patients studied (Figure 1). In all patients the prevalence of K65R in the sequenced viral population was observed at less than $20 \%$, however the prevalence in each individual increased over time. Only in two patients were DRMs to current $1^{\text {st }}$ line therapy observed at prevalence greater than $20 \%$ of the sequenced viral population with G190A and Y181C seen in patients 45 and 76 respectively (Figure 1). In both of these cases the DRM was already present in the patient's viral population prior to antiretroviral (ARV) exposure and was observed to fluctuate during exposure (Figure 1). A similar fluctuating pattern was seen in patient 2 whereby M184V and G190E were not identified by UDPS at baseline or at 14 months yet represented $3 \%$ and $2 \%$ of the sequenced viral population respectively in the sample at 11 months (Figure 1). Aside from DRMs against current $1^{\text {st }}$ line therapy 13 DRMS against other ARVs were identified in four patients (Additional file 2: Figure S2). These mutations were mostly polymorphisms that have limited effect on drug resistance.

\section{Discussion}

We have used ultra deep pyrosequencing (UDPS) to investigate the presence of low abundance drug resistance mutations present within the proviral DNA of individuals on ART. Previous studies have suggested that the mutational archive stored in proviral DNA remains unchanged during HAART [4] with higher levels of DRMs observed in RNA extracted from circulating virus than those present in the provirus [5]. The suggested reasons for these observations are that standard bulk sequencing cannot fully access the spectrum of viral variants stored in the proviral DNA [5] coupled with the fact that resistant variants may be present at low prevalence within the proviral DNA. The sensitivity of UDPS to detect drug resistant minor variants is limited by the number of virus templates that can be successfully extracted and amplified using PCR [12,22]. Through our thorough PCR approach coupled with the use of UDPS as opposed to consensus sequencing we sought to maximise the genetic heterogeneity that was amplified and subsequently sequenced from the proviral DNA. As might be expected, there was an association between PCR amplification success and sequencing success. For patient 32 month 16 , patient 45 month 13 , and the $2^{\text {nd }}$ baseline sample from patient 42, PCR amplification was weaker and thus a higher volume of PCR amplicon had to be added to reach the same final quantity for sequencing as for other samples. For these three samples far more sequences resulting from pyrosequencing had low identity with the consensus sequence and were excluded. It was still worth carrying out pyrosequencing on these samples, however, as thousands of sequences with high identity were returned for analysis. The differences in numbers of quality reads between samples would prevent comparisons of proportions of DRMS across these samples, but not the identification of minor variants.

Bulk sequencing approaches can only detect viral variants present in greater than $20 \%$ of the viral population [23-25] and we did see strong correlation between DRMs observed in bulk sequencing and those at greater than $20 \%$ of the sequenced viral population in the UDPS at the same time-point. There were, however, further DRMs observed at greater than $20 \%$ prevalence that had not been identified using bulk sequencing and the observation of these are likely the result of our approach successfully accessing a greater level of the viral diversity present in the proviral DNA. We would have expected UDPS to detect all of the mutations that were detected via bulk sequencing but this was not the case. From each of five patient samples one DRM, retrieved using the bulk sequencing approach, was absent from the reads retrieved from the samples through the UDPS approach. The phylogenetic origin of each sequence from the bulk sequencing approach was confirmed as belonging to the individual in question, as was the presence of the DRM in the sequence chromatograph indicating that the reporting of the original mutation was not an error, nor was there a mix up of samples. For two samples (patient 32 sample 16 month and baseline 2 samples from patient 42) poor PCR amplification success may be responsible for some mutations being absent. However, for the other three samples this is not the case. Despite utilizing multiple primary and secondary PCRs to maximize the diversity sampled, PCR bottleneck still cannot be excluded as a cause of the absent DRMs. However, as PCR amplifications were from proviral DNA it is 
Table 2 Prevalence of DRMs in pyrosequencing data

\begin{tabular}{|c|c|c|c|c|}
\hline $\begin{array}{l}\text { Patient } \\
\text { number }\end{array}$ & $\begin{array}{l}\text { Sampling time- } \\
\text { point }\end{array}$ & CD4 count & $1-20 \%$ prevalence & $>20 \%$ Prevalence \\
\hline \multirow[t]{3}{*}{ Patient 2} & Baseline & 64 & K65R & \\
\hline & 11 Months & N/A (9 months: 110) & K65R, M184V, G190E & \\
\hline & 14 Months & 153 & K65R & \\
\hline \multirow[t]{4}{*}{ Patient 32} & Baseline & 271 & $\mathrm{~K} 65 \mathrm{R}, \underline{\mathrm{V} 90 \mathrm{I}}$ & \\
\hline & 7 Months & 384 & K65R & $\underline{\text { V90I }}$ \\
\hline & 16 Months & $\begin{array}{l}\text { N/A (19 months: } \\
\text { 443) }\end{array}$ & K65R & \\
\hline & 24 Months & N/A & K65R & \\
\hline \multirow[t]{2}{*}{ Patient 42} & Baseline 1 (2008) & N/A & K65R & \\
\hline & Baseline 2 (2009) & 362 & K65R, A98S, G190A & \\
\hline \multirow[t]{3}{*}{ Patient 45} & Baseline & 55 & $\begin{array}{l}\text { K65R, A98S, K101E, K103E, K103N, K103R, } \\
\text { M184I }\end{array}$ & $\underline{\mathrm{V} 106 \mathrm{I}}, \mathrm{E} 138 \mathrm{~A}, \mathrm{G190 \textrm {A }}$ \\
\hline & 8 Months & 216 & K65R, A98S, K101E, K103E, K103R & V106I, G190A \\
\hline & 13 Months & 258 & K65R, K101E, K103E, K103N, K103R & V1061, E138A, G190A \\
\hline \multirow[t]{3}{*}{ Patient 76} & Baseline & 118 & K65R, T69l, G190A, $\underline{\text { H221Y }}$ & $\underline{\text { V90l, A98S, Y181C }}$ \\
\hline & 6 Months & N/A (8 months: 269) & K65R, $\underline{\mathbf{K} 103 \mathrm{~N}}, \mathrm{H} 221 \mathrm{Y}$ & $\begin{array}{l}\text { V90I, A98S, K103E, Y181C, } \\
\mathrm{H} 221 \mathrm{Y}\end{array}$ \\
\hline & 9 Months & $\begin{array}{l}\text { N/A (12 months: } \\
\text { 268) }\end{array}$ & K65R, V90I, K103E, G190A, $\underline{\mathrm{H} 221 \mathrm{Y}}$ & A98S, K103R, $\underline{\text { Y181C }}$ \\
\hline
\end{tabular}

Only DRMs observed at $>1 \%$ in the sequence data are shown. DRMs relevant to each patient's current therapy regime are shown in bold and those that were also seen in consensus sequencing are underlined. CD4 counts $\left(x 10^{-6} / \mathrm{L}\right)$ are shown. When a CD4 count was not available for a sampling time-point the count from the sample taken at a date closest to that time-point is shown.

perhaps not surprising that some differences would be found between amplicons deriving from different aliquots of DNA, as latent viruses may be more diverse than circulating virus. It should be noted that the presentation of prevalence in our work, as with all other similar studies, should be interpreted as the prevalence observed in the amplified and sequenced viral population as opposed to being a direct measure of the prevalence of a variant in an individual's viral population. The discrepancy between these two interpretations has yet to be elucidated however the exciting development of degenerative primer ID approaches [26] will enable such quantification of these potential biases in the near future and help further resolve the PCR bottle neck issue.

Resistant viruses that make up as little as $1 \%$ of the viral population within an individual have been suggested to be clinically important as they can expand rapidly under the selective pressure exerted by exposure to HAART [10-16]. The ability of UDPS to effectively quantify such variants is limited by PCR and sequencing induced errors. Hedskog and colleagues eloquently showed that the error rate of UDPS is not uniform across sites within the pol gene and that PCR-induced recombination is minimal [27]. Learning from reported issues in other studies we used larger sample volumes [28-30], a high depth of coverage [12,28-30] and a somewhat conservative cutoff of $1 \%$ for identifying low prevalence variants. In all patients we identified low abundance variants with DRMs against the current $1^{\text {st }}$ line therapy. In particular K65R, strongly associated with the development of virologic failure in subtype $\mathrm{C}$ infected individuals [31-37], was observed at low abundance in all patients. While we cannot rule out that the observation of low abundance $\mathrm{K} 65 \mathrm{R}$ at various timepoints in all patients is not as a result of the previously reported propensity for PCR error at this position in subtype $C$ viruses [38] we did use a high fidelity PCR enzyme and multiple primary PCRs to try to avoid such PCR error.

Despite the observation of low abundance drug resistant variants in all individuals, these viruses appear to have had minimal effect on measured treatment outcome. Due to the geographically isolated setting resulting in logistical difficulties viral loads are not routinely assessed and treatment success, to date, has been measured using WHO stages and CD4 counts. Thus, at the final sampling timepoint one (patient 2) is defined as having immune failure (CD4 cell count $<200$ cells $/ \mathrm{mm}^{3}$ after at least 12 months on ART) with all remaining patients showing satisfactory treatment response. While the CD4 counts of two of these individuals, patients 45 and 76, were sufficiently high to avoid being interpreted as treatment failures, their semi-borderline CD4 counts (258 and $268 \mathrm{CD} 4 \mathrm{~T}$ cells cells $/ \mathrm{mm}^{3}$ ) coupled with the 
presence of multiple minor variant DRMs in their provirus could suggest that these patients may be at high risk for imminent failure. Nevertheless, the CD4 counts of all patients rose over the course of the study despite the detection of low abundance variants at early timepoints and all of the participants remained on $1^{\text {st }}$ line therapy until their final sampling timepoint up to as much as 24 months after treatment initiation. Thus, it appears that the presence of low abundance DRMs in the provirus of these individuals has little effect on treatment outcome in the short-term. Previous work suggests that HIV rebounds from latently infected cells rather than as a result of continuing low-level replication [1] and, thus, there is always a possibility that these low abundance resistant variants could emerge to dominate from the proviral DNA following treatment interruption or as a result of poor adherence.

\section{Conclusion}

We observe, in the individuals on continuous antiretroviral therapy studied here, low abundance drug resistant viral variants present in the proviral DNA do not appear to play an immediate role in facilitating the emergence of drug resistance through emergence to dominance. Whether this is true in all instances should be explored further in future longitudinal studies and this group of patients should be monitored further given that in all of the individuals we detected minor variants with DRMs against their current treatment regimen.

\section{Additional files}

Additional file 1: Figure S1. 454 sequence quality control. (A) The tota number of sequenced reads obtained for each sampling time-point is shown as the total size of the bar representing each time-point. The number of reads mapped to the reference sequence and removed as a result of length and identity cutoffs are also shown. (B) The mean and range of read lengths observed for each sequenced sample are shown both before and after quality trimming. In all cases the mean and range of read lengths decreases significantly following trimming.

Additional file 2: Figure S2. Observed DRMs against ARVs not present in the patients' current regimen at all sequenced sampling time-points for each patient. DRMs with observed prevalence less than $1 \%$ of the amplified and sequenced viral population are not included. For each prevalence point, the upper and lower 95\% confidence intervals from the binomial sampling distribution are shown as error bars.

\section{Competing interests}

The authors declare that they have no competing interests.

\section{Authors' contributions}

VB performed the amplification and sequencing of samples and wrote the first draft of the paper. AC, BN, NF and JRG were responsible for the management and running of the Karonga Prevention Study ART project, the collection of samples and all clinical aspects of the project. RKS undertook the analysis examining the effect of sequencing error at homopolymer regions on the calling of drug resistance mutations. GPM supervised the wet-lab work and the interpretation of results while SAT undertook the preliminary bioinformatics analysis of the sequence data and authored subsequent drafts of the paper. All authors contributed to the final draft.

\section{Acknowledgements}

This work was supported by Science Foundation Ireland under Grant No. 07/RFP/EEEOBF424 to Grace McCormack and a grant to Simon Travers from the South African Department of Science and Technology. The Karonga Prevention Study is primarily funded by the Wellcome Trust, Grant No. 079828/Z/06, with contributions from LEPRA. RKS is supported by a PhD student bursary from Atlantic Philanthropies. Permission for the study was received from the National Health Sciences Research Committee, Malawi, and the Ethics Committee of the London School of Hygiene and Tropical Medicine, UK. The authors thank Miguel Lacerda, Natasha Wood and Gordon Harkins for insightful discussions regarding the statistical analysis performed in this manuscript.

\section{Author details}

'Molecular Evolution and Systematics laboratory, Zoology, Ryan Institute, School of Natural Sciences, National University of Ireland Galway, Galway, Ireland. ${ }^{2}$ Karonga Prevention Study, Chilumba, Malawi, South Africa. ${ }^{3}$ Faculty of Epidemiology and Population Health, London School of Hygiene and Tropical Medicine, London, UK. ${ }^{4}$ South African National Bioinformatics Institute, University of the Western, Cape, Bellville, South Africa. ${ }^{5}$ Institute of Infection \& Global Health, University of Liverpool, Liverpool, UK.

Received: 24 May 2012 Accepted: 28 January 2013

Published: 30 January 2013

\section{References}

1. Joos B, Fischer M, Kuster H, Pillai SK, Wong JK, Boni J, Hirschel B, Weber R, Trkola A, Gunthard HF: HIV rebounds from latently infected cells, rather than from continuing low-level replication. Proc Natl Acad Sci U S A 2008, 105(43):16725-16730.

2. Boucher S, Recordon-Pinson P, Neau D, Ragnaud JM, Titier K, Faure M, Fleury H, Masquelier B: Clonal analysis of HIV-1 variants in proviral DNA during treatment interruption in patients with multiple therapy failures. J Clin Virol 2005, 34(4):288-294.

3. Garcia F, Plana M, Vidal C, Cruceta A, O'Brien WA, Pantaleo G, Pumarola T, Gallart T, Miro JM, Gatell JM: Dynamics of viral load rebound and immunological changes after stopping effective antiretroviral therapy. AIDS (London, England) 1999, 13(11):79-86.

4. Palmisano L, Giuliano M, Galluzzo CM, Amici R, Andreotti M, Weimer LE, Pirillo MF, Fragola V, Bucciardini R, Vella S: The mutational archive in proviral DNA does not change during 24 months of continuous or intermittent highly active antiretroviral therapy. HIV Med 2009, 10(8):477-481

5. Wirden M, Soulie C, Valantin MA, Fourati S, Simon A, Lambert-Niclot S, Bonmarchand M, Clavel-Osorio C, Marcelin AG, Katlama C, et al: Historical HIV-RNA resistance test results are more informative than proviral DNA genotyping in cases of suppressed or residual viraemia. J Antimicrob Chemother 2011, 66(4):709-712.

6. Bansode V, Drebert ZJ, Travers SA, Banda E, Molesworth A, Crampin A, Ngwira B, French N, Glynn JR, McCormack GP: Drug resistance mutations in drug-naive HIV type 1 subtype C-infected individuals from rural Malawi. AIDS Res Hum Retrovir 2011, 27(4):439-444.

7. Bansode VB, Travers SA, Crampin AC, Ngwira B, French N, Glynn JR, McCormack GP: Reverse Transcriptase drug resistance mutations in HIV-1 Subtype C infected patients on ART in Karonga District, Malawi. AIDS Res Ther 2011, 8(1):38.

8. Roquebert B, Malet I, Wirden M, Tubiana R, Valantin MA, Simon A, Katlama C, Peytavin G, Calvez V, Marcelin AG: Role of HIV-1 minority populations on resistance mutational pattern evolution and susceptibility to protease inhibitors. AIDS (London, England) 2006, 20(2):287-289.

9. Palmer S, Kearney M, Maldarelli F, Halvas EK, Bixby CJ, Bazmi H, Rock D, Falloon J, Davey RT Jr, Dewar RL, et al: Multiple, linked human immunodeficiency virus type 1 drug resistance mutations in treatmentexperienced patients are missed by standard genotype analysis. J Clin Microbiol 2005, 43(1):406-413.

10. Paredes R, Lalama CM, Ribaudo HJ, Schackman BR, Shikuma C, Giquel F, Meyer WA 3rd, Johnson VA, Fiscus SA, D'Aquila RT, et al: Pre-existing minority drug-resistant HIV-1 variants, adherence, and risk of antiretroviral treatment failure. J Infect Dis 2010, 201(5):662-671.

11. Johnson JA, Li JF, Wei X, Lipscomb J, Irlbeck D, Craig C, Smith A, Bennett $D E$, Monsour M, Sandstrom P, et al: Minority HIV-1 drug resistance 
mutations are present in antiretroviral treatment-naive populations and associate with reduced treatment efficacy. PLoS Med 2008, 5(7):e158.

12. Simen BB, Simons JF, Hullsiek KH, Novak RM, Macarthur RD, Baxter JD, Huang C, Lubeski C, Turenchalk GS, Braverman MS, et al: Low-abundance drug-resistant viral variants in chronically HIV-infected, antiretroviral treatment-naive patients significantly impact treatment outcomes. $J$ Infect Dis 2009, 199(5):693-701.

13. Metzner KJ, Giulieri SG, Knoepfel SA, Rauch P, Burgisser P, Yerly S, Gunthard HF, Cavassini M: Minority quasispecies of drug-resistant HIV-1 that lead to early therapy failure in treatment-naive and -adherent patients. Clin Infect Dis 2009, 48(2):239-247.

14. Kuritzkes DR, Lalama CM, Ribaudo HJ, Marcial M, Meyer WA 3rd, Shikuma C, Johnson VA, Fiscus SA, D'Aquila RT, Schackman BR, et al: Preexisting resistance to nonnucleoside reverse-transcriptase inhibitors predicts virologic failure of an efavirenz-based regimen in treatment-naive HIV-1infected subjects. J Infect Dis 2008, 197(6):867-870

15. Halvas EK, Wiegand A, Boltz VF, Kearney M, Nissley D, Wantman M, Hammer SM, Palmer S, Vaida F, Coffin JM, et al: Low frequency nonnucleoside reverse-transcriptase inhibitor-resistant variants contribute to failure of efavirenz-containing regimens in treatment- experienced patients. J Infect Dis 2010, 201(5):672-680.

16. Palmer S, Boltz V, Maldarelli F, Kearney M, Halvas EK, Rock D, Falloon J, Davey RT Jr, Dewar RL, Metcalf JA, et al: Selection and persistence of nonnucleoside reverse transcriptase inhibitor-resistant HIV-1 in patients starting and stopping non-nucleoside therapy. AIDS (London, England) 2006, 20(5):701-710.

17. Jakobsen MR, Tolstrup M, Sogaard OS, Jorgensen LB, Gorry PR, Laursen A, Ostergaard L: Transmission of HIV-1 drug-resistant variants: prevalence and effect on treatment outcome. Clin Infect Dis 2010, 50(4):566-573.

18. Peuchant $O$, Thiebaut $R$, Capdepont S, Lavignolle-Aurillac V, Neau D, Morlat P, Dabis F, Fleury H, Masquelier B: Transmission of HIV-1 minority-resistant variants and response to first-line antiretroviral therapy. AIDS (London, England) 2008, 22(12):1417-1423.

19. Drummond AJ AB, Buxton S, Cheung M, Cooper A, Heled J, Kearse M, Moir R, Stones-Havas S, Sturrock S, Thierer T, Wilson A: Geneious v5.1. 2010. Available from http://www.geneious.com.

20. Archer J, Rambaut A, Taillon BE, Harrigan PR, Lewis M, Robertson DL: The evolutionary analysis of emerging low frequency HIV-1 CXCR4 using variants through time-an ultra-deep approach. PLoS Comput Biol 2010, 6(12):e1001022

21. Dudley DM, Chin EN, Bimber BN, Sanabani SS, Tarosso LF, Costa PR, Sauer MM, Kallas EG, O'Connor DH: Low-cost ultra-wide genotyping using Roche/454 pyrosequencing for surveillance of HIV drug resistance. PLoS One 2012 7(5):e36494.

22. Shafer RW: Low-abundance drug-resistant HIV-1 variants: finding significance in an era of abundant diagnostic and therapeutic options. J Infect Dis 2009, 199(5):610-612.

23. Korn K, Reil H, Walter H, Schmidt B: Quality control trial for human immunodeficiency virus type 1 drug resistance testing using clinical samples reveals problems with detecting minority species and interpretation of test results. J Clin Microbiol 2003, 41(8):3559-3565.

24. Gunthard HF, Wong JK, Ignacio CC, Havlir DV, Richman DD: Comparative performance of high-density oligonucleotide sequencing and dideoxynucleotide sequencing of HIV type 1 pol from clinical samples. AIDS Res Hum Retrovir 1998, 14(10):869-876.

25. Van Laethem K, Van Vaerenbergh K, Schmit JC, Sprecher S, Hermans $P$, De Vroey V, Schuurman R, Harrer T, Witvrouw M, Van Wijngaerden $E_{\text {, et }}$ al: Phenotypic assays and sequencing are less sensitive than point mutation assays for detection of resistance in mixed HIV-1 genotypic populations. J Acquir Immune Defic Syndr 1999, 22(2):107-118.

26. Jabara CB, Jones CD, Roach J, Anderson JA, Swanstrom R: Accurate sampling and deep sequencing of the HIV-1 protease gene using a Primer ID. Proc Natl Acad Sci U S A 2011, 108(50):20166-20171.

27. Hedskog C, Mild M, Jernberg J, Sherwood E, Bratt G, Leitner T, Lundeberg J, Andersson B, Albert J: Dynamics of HIV-1 quasispecies during antiviral treatment dissected using ultra-deep pyrosequencing. PLoS One 2010, 5(7):e11345

28. Wang C, Mitsuya Y, Gharizadeh B, Ronaghi M, Shafer RW: Characterization of mutation spectra with ultra-deep pyrosequencing: application to HIV1 drug resistance. Genome research 2007, 17(8):1195-1201.
29. Varghese $V$, Shahriar R, Rhee SY, Liu T, Simen BB, Egholm M, Hanczaruk B, Blake LA, Gharizadeh B, Babrzadeh F, et al: Minority variants associated with transmitted and acquired HIV-1 nonnucleoside reverse transcriptase inhibitor resistance: implications for the use of secondgeneration nonnucleoside reverse transcriptase inhibitors. J Acquir Immune Defic Syndr (1999) 2009, 52(3):309-315.

30. Le T, Chiarella J, Simen BB, Hanczaruk B, Egholm M, Landry ML, Dieckhaus K, Rosen MI, Kozal MJ: Low-abundance HIV drug-resistant viral variants in treatment-experienced persons correlate with historical antiretroviral use. PLoS One 2009, 4(6):e6079.

31. Doualla-Bell F, Avalos A, Brenner B, Gaolathe T, Mine M, Gaseitsiwe S, Oliveira M, Moisi D, Ndwapi N, Moffat H, et al: High prevalence of the K65R mutation in human immunodeficiency virus type 1 subtype $C$ isolates from infected patients in Botswana treated with didanosine-based regimens. Antimicrob Agents Chemother 2006, 50(12):4182-4185.

32. Hosseinipour MC, van Oosterhout JJ, Weigel R, Phiri S, Kamwendo D, Parkin N, Fiscus SA, Nelson JA, Eron JJ, Kumwenda J: The public health approach to identify antiretroviral therapy failure: high-level nucleoside reverse transcriptase inhibitor resistance among Malawians failing first-line antiretroviral therapy. AIDS (London, England) 2009, 23(9):1127-1134.

33. Brenner BG, Oliveira M, Doualla-Bell F, Moisi DD, Ntemgwa M, Frankel F, Essex M, Wainberg MA: HIV-1 subtype C viruses rapidly develop K65R resistance to tenofovir in cell culture. AIDS (London, England) 2006, 20(9):9-13.

34. Coutsinos D, Invernizzi CF, Moisi D, Oliveira M, Martinez-Cajas JL, Brenner BG, Wainberg MA: A template-dependent dislocation mechanism potentiates $\mathrm{K} 65 \mathrm{R}$ reverse transcriptase mutation development in subtype C variants of HIV-1. PloS one 2011, 6(5):e20208.

35. Coutsinos D, Invernizzi CF, Xu H, Moisi D, Oliveira M, Brenner BG, Wainberg $M A$ : Template usage is responsible for the preferential acquisition of the K65R reverse transcriptase mutation in subtype $C$ variants of human immunodeficiency virus type 1. J Virol 2009, 83(4):2029-2033.

36. Invernizzi CF, Coutsinos D, Oliveira M, Moisi D, Brenner BG, Wainberg MA: Signature nucleotide polymorphisms at positions 64 and 65 in reverse transcriptase favor the selection of the K65R resistance mutation in HIV1 subtype C. J Infect Dis 2009, 200(8):1202-1206.

37. Sunpath H, Wu B, Gordon M, Hampton J, Johnson B, Moosa MY, Ordonez C, Kuritzkes DR, Marconi VC: High rate of K65R for antiretroviral therapynaive patients with subtype C HIV infection failing a tenofovircontaining first-line regimen. AIDS 2012, 26(13):1679-1684.

38. Varghese V, Wang E, Babrzadeh F, Bachmann MH, Shahriar R, Liu T, Mappala SJ, Gharizadeh B, Fessel WJ, Katzenstein D, et al: Nucleic acid template and the risk of a PCR-Induced HIV-1 drug resistance mutation. PloS one 2010, 5(6):e10992.

doi:10.1186/1471-2334-13-52

Cite this article as: Bansode et al: Characterizing the emergence and persistence of drug resistant mutations in HIV-1 subtype $\mathrm{C}$ infections using 454 ultra deep pyrosequencing. BMC Infectious Diseases 2013 13:52.

\section{Submit your next manuscript to BioMed Central and take full advantage of:}

- Convenient online submission

- Thorough peer review

- No space constraints or color figure charges

- Immediate publication on acceptance

- Inclusion in PubMed, CAS, Scopus and Google Scholar

- Research which is freely available for redistribution 